\title{
Bullshit Jobs
}

\section{Erhard Taverna}

Dr. med., Mitglied der Redaktion

Im Schweisse deines Angesichts sollst du dein Brot essen, sagt uns die Bibel. «Syt dihr öpper oder nähmet dihr Lohn» lautet eine der unzähligen Anekdoten, die von Madame de Meuron erzählt werden. Das stadtbekannte Original aus dem Berner Patriziat vertrat seine feudale Definition von Arbeit, die jahrhundertelang gültig war. Karl Marx glaubte, dass es nach dem Sieg des Kommunismus jedem möglich sein werde, «morgens zu jagen, nachmittags zu fischen, abends Viehzucht zu treiben und nach dem Essen zu kritisieren". Eine Freizeitgesellschaft, die der britische Ökonom Keynes (1883-1945) als das grösste Problem für die Menschen im Jahre 2030 betrachtete. Dank hoher Produktivität werde eine 15-Stunden-Woche zum Leben ausreichen. Teilzeitarbeit oder die 40-Stunden-Woche ist seither für viele eine Realität, Segen oder Fluch, je nachdem, wer dazu befragt wird.

\section{Ein Job, den niemand vermisst, wenn es ihn nicht geben würde.}

Der amerikanische Anthropologe David Graeber, Lehrer an der London School of Economics, provoziert mit seiner Definition des Bullshit Job. Ein Job, den niemand vermisst, wenn es ihn nicht geben würde. Jeder zweite, vor allem im Büro, sei völlig sinnlos, behauptet er. Zwar würden neue Technologien schon heute die Voraussage von Keynes ermöglichen, doch seien, gemäss seinen Recherchen, nur viele neue, überflüssige und frustrierende Jobs entstanden. Er nennt Immobilienmakler, Unternehmensberater und Investmentbanker die Hofnarren des Kapitalismus. Niemand würde sie vermissen, wenn es sie nicht mehr gäbe. Ebenso wie unzählige neue Tätigkeiten, etwa Kultur- und Eventmanager, Fachreferenten für medizinisches Versorgungswesen oder Regionalkoordinatoren im Bildungsmarketing. Die Liste lässt sich beliebig verlängern. Graeber begnügt sich mit einer relativ kleinen Zahl von Stichproben. Gibt es einen zumindest statistisch gesicherten Massstab für den Nutzen einer Arbeit? Das Institut für Demoskopie Allensbach befragt die Bevölkerung regelmässig, welche Berufe sie am meisten schätzt. Das grösste Berufsprestige geniessen seit Jahren Ärztinnen, Krankenschwestern, Polizisten und
Lehrer. Es sind die klar umrissenen Berufsbilder, die viele spezialisierte Berufe unerwähnt lassen, da deren gesellschaftlicher Nutzen von aussen schwierig einzuschätzen ist. Der Deutsche Gewerkschaftsbund ermittelt immer wieder, wie sinnvoll Arbeitnehmer ihren Job empfinden. Rund ein Drittel hat den Eindruck, dass seine Arbeit keinen gesellschaftlichen Nutzen abwerfe, aber weit mehr als die Hälfte davon ist trotzdem zufrieden. Das subjektive Sinnerleben deckt sich nicht unbedingt mit dem, was aussenstehende Kritiker vermuten. Man kann ganz anders als Graeber argumentieren und darauf hinweisen, dass in einer extrem arbeitsteiligen Gesellschaft auch die Illusion von Arbeit einen Zusammenhalt ergibt, eine Sicherheit gegen die Launen des Marktes und die Ungewissheiten der Automatisierung. Arbeit ist ein Teil der Identität. Wer seine Anstellung verliert, wird bei einer Neueinstellung kaum den sozialen Nutzen seiner Anstellung hinterfragen, zumindest solange der Lohn stimmt. Im Zeitalter der Digitalisierung wird der Fürsorgecharakter von Arbeit zunehmen, so eine These des Autors. Dazu gehörten auch Nachbarschaftshilfe, Eltern- und Grosselternaufgaben und viele Ehrenämter. Die Arbeit gehe nicht aus, sie müsse nur anders finanziert werden.

David Graeber hat 2011 aktiv an der Occupy Wall StreetProtestbewegung mitgewirkt. In Zürich folgte die Occupy Paradeplatz vor dem Hauptsitz der UBS und Credit Suisse. Es war die Zeit grosser Hoffnungen, wie die des Arabischen Frühlings. Graeber ist für das Experiment eines bedingungslosen Grundeinkommens. Die wenigsten wollten auf der faulen Haut liegen, genauso gut könnten ganz neue kreative Tätigkeiten entstehen.

Am 5. Juni 2016 haben die Stimmberechtigten in der Schweiz, als erstem Land, über ein bedingungsloses Grundeinkommen abgestimmt. Die Initiative wurde mit 76,9\% Nein-Stimmen verworfen. Bullshit Jobs hin oder her, die Arbeitswelt ändert sich rasant. Es dürfte nicht die letzte Abstimmung zu diesem Thema gewesen sein.

Literatur

David Graeber. Bullshit-Jobs. Klett-Cotta Verlag; 2018. 